\title{
CARMENES: Calar Alto high-Resolution search for $M$ dwarfs with Exo-earths with Near-infrared and optical Echelle Spectrographs
}

\author{
Andreas Quirrenbach ${ }^{1}$, Pedro J. Amado ${ }^{2}$, José A. Caballero ${ }^{3}$, \\ Holger Mandel ${ }^{1}$, Reinhard Mundt ${ }^{4}$, Ansgar Reiners ${ }^{5}$, Ignasi Ribas ${ }^{6}$, \\ Miguel A. Sánchez Carrasco ${ }^{2}$, Walter Seifert ${ }^{1}$ \\ and the CARMENES Consortium ${ }^{1,2,3,4,5,6,7,8,9,10,11}$ \\ ${ }^{1}$ Landessternwarte Königstuhl, Heidelberg, Germany \\ email: A.Quirrenbach@lsw. uni-heidelberg.de \\ ${ }^{2}$ Instituto de Astrofísica de Andalucía, Granada, Spain \\ ${ }^{3}$ Centro de Astrobiología, Madrid, Spain \\ ${ }^{4}$ Max-Planck-Institut für Astronomie, Heidelberg, Germany \\ ${ }^{5}$ Insitut für Astrophysik, Göttingen, Germany \\ ${ }^{6}$ Institut de Ciències de l'Espai, Barcelona, Spain \\ ${ }^{7}$ Instituto de Astrofísica de Canarias, Tenerife, Spain \\ ${ }^{8}$ Thüringer Landessternwarte, Tautenburg, Germany \\ ${ }^{9}$ Hamburger Sternwarte, Hamburg, GermanY \\ ${ }^{10}$ Universidad Complutense de Madrid, Madrid, Spain \\ ${ }^{11}$ Centro Astronómico Hispano-Alemán, Almería, Spain
}

\begin{abstract}
CARMENES (Calar Alto high-Resolution search for $\mathbf{M}$ dwarfs with Exo-earths with Near-infrared and optical Echelle Spectrographs) is a next-generation instrument for the $3.5 \mathrm{~m}$ telescope at the Calar Alto Observatory. CARMENES will conduct a five-year exoplanet survey targeting $\sim 300 \mathrm{M}$ stars. The CARMENES instrument consists of two separate fiber-fed spectrographs covering the wavelength range from 0.52 to $1.7 \mu \mathrm{m}$ at a spectral resolution of $R=$ 85,000 . The spectrographs are housed in a temperature-stabilized environment in vacuum tanks, to enable a $1 \mathrm{~m} / \mathrm{s}$ radial velocity precision employing a simultaneous emission-line calibration.
\end{abstract}

Keywords. instrumentation: spectrographs, techniques: radial velocities, infrared: planetary systems

\section{CARMENES Science}

he aim of CARMENES is to perform high-precision measurements of stellar radial velocities with long-term stability. The fundamental science objective is to carry out a survey of late-type main sequence stars (with special focus on moderately active stars of spectral type $\mathrm{M} 4 \mathrm{~V}$ and later) with the goal of detecting low-mass planets in their habitable zones. For stars later than M4-M5 $\left(M<0.20 \mathrm{M}_{\odot}\right)$, a radial velocity precision of $1 \mathrm{~m} \mathrm{~s}^{-1}$ (per measurement; $\sigma_{i}$ ) will permit the detection of super-Earths of $5 \mathrm{M}_{\oplus}$ and smaller inside the entire width of the habitable zone with $2 \sigma_{i}$ radial-velocity amplitudes (i.e., $K_{p}=2 \mathrm{~m} \mathrm{~s}^{-1}$ ). For a star near the hydrogen-burning limit and a precision of $1 \mathrm{~m} \mathrm{~s}^{-1}$, a planet as small as our own Earth in the habitable zone could be detected. In addition, the habitable zones of all M-type dwarfs can be probed for super-Earths. 
The CARMENES survey will be carried out with the $3.5 \mathrm{~m}$ telescope on Calar Alto, using at least 600 clear nights in the 2014-2018 time frame. We plan to survey a sample of 300 M-type stars for low-mass planet companions. This will provide sufficient statistics to assess the overall distribution of planets around $M$ dwarfs: frequency, masses, and orbital parameters. The seemingly low occurrence of Jovian planets should be confirmed, and the frequency of ice giants and terrestrial planets should be established along with their typical separations, eccentricities, multiplicities, and dynamics.

\section{The CARMENES Spectrographs}

In order to identify the wavelength range that is most suitable for the search for radial velocity variations in low-mass stars, we have carried out detailed simulations of the achievable precision of such a measurement (see also Reiners et al. 2010). To optimize both the radial-velocity precision and the ability to discriminate between (wavelengthdependent) intrinsic radial-velocity jitter and (wavelength-independent) Keplerian signals, we decided to build two separate spectrographs, which will cover the wavelength ranges from 0.52 to $1.05 \mu \mathrm{m}$ and 1.0 to $1.7 \mu \mathrm{m}$, respectively.

To take full advantage of the information content in a stellar spectrum for radial velocity measurements, the spectrograph resolution has to be matched to the stellar line width. Since many of the potential targets for CARMENES have rotational velocities of $3 \mathrm{~km} \mathrm{~s}^{-1}$ or less, a resolution of up to $\sim 100,000$ could be usefully exploited. As a compromise between cost, size, and complexity on one hand, and performance on the other hand, the CARMENES spectrographs will have $R \sim 85,000$, realized through cross-dispersed echelle formats with white-pupil designs (for details see Quirrenbach et al. 2010). The visible-light spectrograph will use a $2048 \times 4096$ pixel CCD; the near infrared spectrograph will employ a mosaic of two $2048 \times 2048$ pixel detectors. To ensure sufficient stability, both spectrographs will be housed in temperature-stabilized vacuum tanks.

The spectrographs will be coupled to the $3.5 \mathrm{~m}$ telescope with optical fibers. Because there is currently no known gas cell that gives a sufficiently dense grid of absorption lines over the whole bands used by CARMENES, the simultaneous emission line method, using ThAr or alternatively UNe lamps, has been adopted as a baseline for the precise wavelength calibration. A stabilized etalon is currently under investigation as a potential alternative.

\section{References}

Quirrenbach, A., et al. 2010, in: Ground-based and airborne instrumentation for astronomy III, Eds. McLean, I.S., Ramsay, S.K., \& Takami, H., Proc. SPIE, 7735, 1

Reiners, A., et al. 2010,ApJ, 710, 432 